\title{
The Timed Up-\&-Go Test: Preliminary Age- and Sex-related Reference Values for Older Filipino Adults
}

\author{
Edward James R. Gorgon \\ Department of Physical Therapy, College of Allied Medical Professions, University of the Philippines Manila
}

\begin{abstract}
Objective. The Timed Up-\&-Go Test (TUGT) is a clinically useful measure that has been widely used in practice to assess functional mobility in older people. Interpretation of TUGT scores relies on appropriate reference values. This study aimed to describe preliminary age- and sex-related reference values for the TUGT for Filipinos aged 60-79 years.
\end{abstract}

Methods. This is a descriptive cross-sectional study. We included Filipino adults aged 60-79 years, with no significant disability, and resided in metropolitan areas in the National Capital Region and rural communities in southern Luzon. All participants completed the TUGT. Data were analyzed descriptively and reported as means, standard deviations, and $95 \%$ confidence intervals.

Results. A total of 156 community-dwelling older adults participated in the study with mean age (SD) of 68 (5) years. The majority were women (103/156, 66\%). Mean (SD) TUGT score for all participants was 11.0 (2.4) seconds. Overall, men completed the test faster compared to women, and individuals in the 60-69 years age group had shorter completion times than those in the 70-79 years age group.

Conclusion. In the absence of definitive reference values for older Filipino adults, this study provides preliminary guidance for interpreting TUGT performance for the purposes of screening and monitoring functional mobility impairments in this population.

Key Words: aging, developing countries, functional mobility, geriatric assessment, normative data, rehabilitation

\section{INTRODUCTION}

Corresponding author: Edward James R. Gorgon, MPhysio Department of Physical Therapy College of Allied Medical Professions University of the Philippines Manila Pedro Gil Street, Ermita, Manila 1000, Philippines Email: ergorgon@up.edu.ph
Mobility refers to an individual's ability to change body position or move from one place to another in the environment in an independent and safe manner. ${ }^{1-2}$ Independent mobility is critical to healthy aging and quality of life. ${ }^{3}$ Impairments in mobility lead to significant disability and increased mortality risk in older people. ${ }^{4}$ Mobility changes occur with aging as the body undergoes normal physiologic changes. ${ }^{5}$ Older people experience mobility limitations more than any age group and such limitations typically represent the first obvious sign of functional decline. ${ }^{6}$ It is therefore crucial to screen and monitor mobility limitations in older people in order to design appropriate and timely interventions.

The Timed Up-\&-Go Test (TUGT) is a clinically applicable measure that has been used to assess functional mobility, balance, and risk of falls in older people. ${ }^{7}$ The TUGT was originally developed to monitor basic mobility skills (i.e., standing up and sitting down, walking over a short distance, and turning while walking) in frail community- 
dwelling older people. ${ }^{8}$ It has since been used in various patient populations and recommended as a core outcome measure for neurologic conditions such as stroke, traumatic brain injury, Parkinson's disease, multiple sclerosis, spinal cord injury, and vestibular disorders. ${ }^{9}$ The wide clinical application of the TUGT is due to the high practicality of test administration: it is easy to set up in various practice settings (i.e., space and instrumentation requirements are little); is quick to complete (administration time is less than 3 minutes); involves no additional cost; and requires no specialized assessor training. ${ }^{7}$

Interpretation of TUGT scores is norm-referenced, which means that scores are compared with aggregated test scores of a reference or "normal" group ${ }^{10}$ (i.e., people with no known disability and who are of similar age, biological sex, and anthropometric features). The influence of age and sex on TUGT performance of healthy adults has been demonstrated. ${ }^{11}$ Findings of various studies reporting TUGT reference values have been pooled in a descriptive meta-analysis and reported by decade of life ${ }^{12}$ however, most of the included studies were from Western populations and scores were not reported separately for men and women per age group. Studies that have reported sex-related reference values per decade of life used populations from Western countries and/or included older people who were dependent on walking aids. ${ }^{13-14}$

Populations in the Southeast Asia (SEA) region have anthropometric characteristics that are affected by their unique diet, habitat, nature of work, and geographical location. ${ }^{15}$ Even among the geographically-related populations of Indonesia, Thailand, Malaysia, and the Philippines, differences in anthropometric characteristics have been described. For example, one study comparing available cohort data showed that Filipinos have the shortest mean height compared to Indonesians, Thai, and Malaysians. ${ }^{16}$ Height is a factor that influences walking speed and differences in height may impact the walking component of the TUGT. To date, only one study has reported both age- and sex-related TUGT reference values for older people in a SEA country (Thailand). ${ }^{15}$ The TUGT reference values generated from this SEA cohort demonstrated important differences from those described in a similar study using a North American cohort, ${ }^{13}$ thus supporting the need for population-specific data. This study aimed to describe preliminary age- and sexrelated reference values for the TUGT from a sample of older Filipino adults living in urban and rural communities.

\section{MATERIALS AND METHODS}

\section{Study Design}

This study used a descriptive study design. ${ }^{10}$ The Ethical Review Committee of the University of the Philippines Manila - College of Allied Medical Professions approved the study protocol. All participants provided written informed consent.

\section{Study Participants}

Participants residing in urban communities in the National Capital Region and rural communities in southern Luzon provinces (i.e., Rizal, Cavite, and Laguna) were recruited through convenience sampling. Potential participants were invited through public announcements and active recruitment in senior citizen organizations. Eligibility criteria for the study participants are detailed in Table 1. Briefly, participants were Filipino citizens who were at least 60 years of age, had no mobility disability, and had no health condition that precluded feasible and safe testing. Mobility disability was defined as inability to walk at least one half mile (800 meters) and/or ascend and descend at least one flight of stairs without supervision for safety or physical assistance from at least one person. ${ }^{17}$

\section{Materials and Instruments}

Data on participant eligibility and demographic characteristics were collected using a standardized form for the study. Height and weight were measured using a measuring tape mounted on a wall and portable weighing scale, respectively. Materials and physical set-up for the TUGT as originally described by Podsiadlo and Richardson was used: a standard armchair (approximate seat height $=$ 46 centimeters), three-meter walking distance on the floor, visible floor marker to indicate the point for turning, and digital stopwatch to measure time. ${ }^{8}$ The author trained all assessors on participant screening and TUGT administration. Assessors rehearsed the TUGT procedure under supervision by the author, and using the correct test set-up and a printed

Table 1. Participant inclusion and exclusion criteria

\begin{tabular}{|c|c|}
\hline Inclusion criteria & Exclusion criteria \\
\hline Aged 60 years and older & Required an assistive device in standing or walking \\
\hline $\begin{array}{l}\text { Community-dwelling (i.e., not residing in short- or long-term } \\
\text { care home) }\end{array}$ & $\begin{array}{l}\text { Had a significant medical condition affecting standing balance } \\
\text { and mobility, such as a major surgery or neuromusculoskeletal }\end{array}$ \\
\hline Independent in basic activities of daily living & injury or disease within the past six months, loss of consciousness \\
\hline $\begin{array}{l}\text { Could walk outdoors at least one half mile ( } 800 \text { meters) without } \\
\text { supervision or assistance* }\end{array}$ & $\begin{array}{l}\text { or falling within the previous three months, or uncorrected } \\
\text { visual impairment }\end{array}$ \\
\hline $\begin{array}{l}\text { 5. Could climb up and down at least one flight of stairs without } \\
\text { supervision or assistance* }\end{array}$ & $\begin{array}{l}\text { Had a health condition that precluded safe mobility testing, } \\
\text { such as uncontrolled hypertension or unstable angina, or an }\end{array}$ \\
\hline Could follow simple instructions in English or Filipino & acute illness or injury at the time of testing \\
\hline 7. Could provide informed consent & Unable to follow three-level instructions \\
\hline
\end{tabular}

${ }^{*}$ Inability to meet this minimum requirement has been used to define mobility disability ${ }^{17}$ 

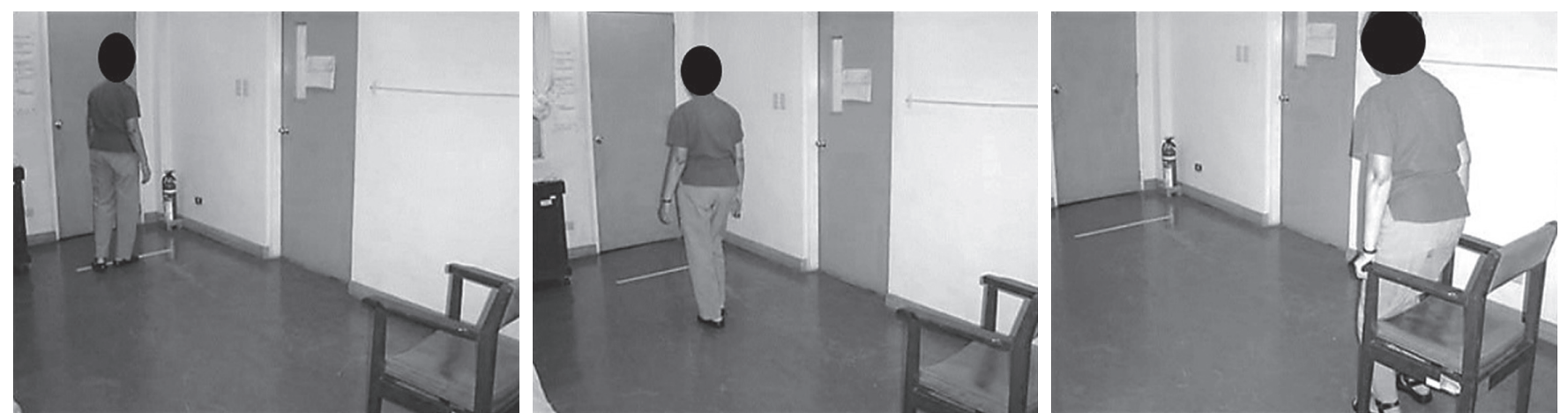

Figure 1. The Timed Up-\&-Go Test: On the word "Go", the participant rises from a standard armchair, walks three meters, turns around at the line marker on the floor, walks back to the armchair, then sits down. The time taken to complete the test (in seconds) is recorded as the score.

script containing the standardized test instructions. All assessors were previously familiar with the TUGT as they had completed a formal clinical assessment course as part of their undergraduate physical therapy training.

\section{Study Procedure}

Assessors screened participants for eligibility and carried out data collection in community areas with sufficient ventilation, adequate lighting, and minimal distractions (e.g., community or church multi-purpose halls or function rooms, or the participant's home). Testing was scheduled in the morning to minimize diurnal variations in physical performance. Participants wore comfortable clothing and their usual footwear.

Assessors administered the TUGT based on the original procedure described by (Figure 1). ${ }^{8}$ Time (in seconds) was measured in completing this series of tasks: standing up from an armchair, walking over a three-meter walkway, turning at a floor marker at the end of three meters, walking back to the armchair, and sitting down again. Each participant started with the back against the chair and proceeded to get up to walk toward the floor marker and return to the chair upon the assessor's command "go." Timing was stopped once the participant had sat again (i.e., once the buttocks had made contact with the chair). Participants walked at their usual or comfortable speed. Assessors provided verbal instructions in either Filipino or English, whichever language the participants preferred, and demonstrated the procedure. One practice trial was provided to allow the participants to familiarize with the procedure and two test trials were conducted. Average scores were used in data analysis.

\section{Data Analysis}

All data were anonymized to ensure confidentiality and analyzed descriptively using IBM $^{\circledR}$ SPSS $^{\circledR}$ Statistics (Version 24, IBM Corporation, New York, USA). Categorical data were reported as frequencies and percentages. Continuous data demonstrated normal distribution based on both visual inspection of histograms and the Kolmogorov-Smirnov Test $(\mathrm{P}>0.05)$. Thus, continuous data were reported as means, standard deviations, and 95\% confidence intervals. Performance on the TUGT was reported by age (i.e., 60-69 years and 70-79 years) and biological sex, to allow for crosscomparisons with published data from similar studies. ${ }^{13,15}$

\section{RESULTS}

One hundred fifty six older adults with mean (SD) age of 68 (5) years, $66 \%$ of whom were women, participated in the study. Mean (SD) height and weight were $1.56(0.08)$ meters and 52.9 (7.9) kilograms, respectively. A similar number of older adults from urban and rural communities participated ( $\mathrm{n}=86$ and 70 , respectively).

Demographic characteristics of participants (mean age, height, and weight) in each group determined by age and sex (i.e., 60-69 years, men; 60-69 years, women; 70-79 years, men; 70-79 years, women), and age- and sex-related reference values for the TUGT are detailed in Table 2 .

Table 2. Participant characteristics and age- and sex-related TUGT performance

\begin{tabular}{lcccccc} 
Participants & No. & Mean (SD) age (yrs.) & Mean (SD) height (m) & Mean (SD) weight (kg) & Mean (SD) TUGT score (s) & 95\% CI (s) \\
$\begin{array}{lccccc}\text { 60-69 years } \\
\text { Men }\end{array}$ & & & & & \\
Women & 64 & $64.8(2.8)$ & $1.67(0.08)$ & $63.1(11.6)$ & $10.3(2.0)$ & $9.5-11.0$ \\
\hline 70-79 years & $64.5(2.6)$ & $1.54(0.04)$ & $50.7(5.0)$ & $11.0(2.4)$ & $10.4-11.6$ \\
Men & 20 & $73.7(3.9)$ & $1.61(0.03$ & $53.5(3.8)$ & $10.7(2.2)$ & $9.6-11.7$ \\
Women & 40 & $74.2(2.6)$ & $1.55(0.06)$ & $49.8(2.2)$ & $11.8(2.6)$ & $10.9-12.6$ \\
\hline
\end{tabular}

Note: SD, Standard deviation; $\mathrm{Cl}$, confidence interval; TUGT, Timed Up-\&-Go Test 
Overall, TUGT completion times were shorter for younger compared with older age groups regardless of sex. Similarly, TUGT completion times were shorter for men compared with women from the same age group. In addition, urbandwelling participants $(n=86)$ completed the TUGT faster than their rural-dwelling counterparts $(\mathrm{n}=70)$ : mean (SD) 10.1 (2.0) seconds (95\% CI 9.7-10.5) versus 12.2 (2.3) seconds (95\% CI 11.6-12.7), respectively.

\section{DISCUSSION}

This study is the first to report age- and sex-related TUGT reference values for older Filipino adults. Key findings align with those of published studies that TUGT completion times generally demonstrate age-related changes and are generally shorter (faster) for men than women. ${ }^{12-13,15,18}$ The effects of age- and sex-related functional decline in body systems have been suggested to explain differences in mobility test performance across age groups and between men and women. ${ }^{15}$ Height and weight can also be significant contributors to the performance of older people on functional mobility tests. ${ }^{15}$ Mean TUGT scores across the different groups in this study differed (i.e., were approximately 2-3 seconds longer or slower) from those in a similar study among North Americans who were approximately taller by $5-11$ centimeters and heavier by 20-29 kilograms. ${ }^{13}$ Findings from this study underscore the need to generate population-specific (i.e., Filipino-specific) reference values for functional mobility tests, including the TUGT.

Mean TUGT scores of the study participants were more similar to those reported for a large sample of older Thai adults $(n=1,030)$ by Thaweewannakij, et al. ${ }^{15}$ Across the age- and sex-related groups in the Thai study, TUGT mean scores were: 9.2 seconds for 60- to 69-year-old males; 9.9 seconds for 60- to 69-year-old females; 10.2 seconds for 70to 79-year-old males; and 11.3 seconds for 70- to 79-year-old females. One key difference between studies was that, given the large sample size employed in the Thai study, it was more likely to have estimated the reference values with greater precision. However, the Thai study recruited participants almost entirely from rural and semi-rural communities, ${ }^{19}$ while the sample for the present study comprised older people from both urban and rural communities.

Generalizability of the findings of this study could be enhanced by its inclusion of participants from both urban and rural settings. Previous studies reporting both age- and sex-related TUGT reference values had involved samples from primarily either metropolitan ${ }^{13}$ or rural and semi-rural ${ }^{15}$ settings only. In this study, urban-dwelling participants completed the TUGT faster than their ruraldwelling counterparts. This apparent difference in mobility test performance between urban-dwelling and ruraldwelling cohorts is consistent with findings from a study of 180 older Filipinos for the Five Times Sit-to-Stand
Test, 10 Meter Walk Test, and Six Minute Walk Test. ${ }^{19}$ In part, this finding may be explained by the higher functional status among urban-dwelling older Filipino adults. ${ }^{20}$ The hypothesis that residential setting determines mobility test performance in older people requires further research using an appropriate study design.

Given the overall dearth of reference values for functional mobility tests for older Filipino adults, the findings of this study may provide useful preliminary information on which future normative studies can build. It can provide preliminary guidance on the interpretation of TUGT scores in local clinical practice. For example, available data from a Belgian cohort indicate that older adults should be expected to complete the TUGT in 10 seconds or less; ${ }^{11}$ however, data from this study suggest that older Filipino adults who do not have a disability may complete it in 10-12 seconds, on average. Greater contextualization in the interpretation of TUGT reference values is important, as this test remains widely used. Its wide use is due to its ability to monitor functional mobility in older people with or without disability, low likelihood of demonstrating ceiling effects, and association with executive function. ${ }^{21}$

The findings of this study need to be interpreted in view of the limitations. Use of convenience sampling limits generalization of findings to the broader older Filipino adult population. Sample sizes across the different participant subgroups were uneven, with a greater proportion of participants included in the 60-69 years age groups and female groups. In addition, no data were reported for people aged 80 years and older. Furthermore, the sample did not allow analysis of the age- and sex-related reference values based on other potentially relevant factors such as residential setting. Therefore, the study results should be interpreted as preliminary reference values, until a definitive study with a stronger sampling strategy has been carried out.

\section{CONCLUSION}

In summary, this study provides preliminary reference values for the TUGT for older Filipino adults which can potentially provide greater contextualization of test interpretation in local clinical practice and serve as baseline data on which future normative studies can build. Future studies should aim to establish definitive normative data through a stronger sampling strategy (e.g., more balanced sample sizes across participant groups and representation of individuals who are aged 80 years and over). The potential effect of residential setting on mobility performance needs to be explored given its implication on test interpretation.

\section{Acknowledgments}

The author acknowledges the contribution of the following individuals in data collection: Giana Balbuena, Gelyn Dimaculangan, Erika Feliciano, Abigail Galang, Carlo Legaspi, Gerald Libranda, Sheigne Miñano, Katrina 
Osana, and Eidref Santos. The author also thanks Alma Labro for her assistance in project management.

\section{Statement of Authorship}

The author conceived and designed the study; organized data collection; analyzed and interpreted the data; and wrote the manuscript for publication.

\section{Author Disclosure}

The author declares no conflict of interest.

\section{Funding Source}

No funding was received for this study.

\section{REFERENCES}

1. Soubra R, Chkeir A, Novella JL. A systematic review of thirty-one assessment tests to evaluate mobility in older adults. BioMed Res Int. 2019: 1354362.

2. Gorgon E, Said C, Galea M. Mobility on discharge from an aged care unit. Physiother Res Int. 2007 Jun; 12(2):72-81.

3. Webber SC, Porter MM, Menec VH. Mobility in older adults: A comprehensive framework. Gerontologist. 2010 Aug; 50(4):443-50.

4. Hirvensalo M, Rantanen T, Heikkinen E. Mobility difficulties and physical activity as predictors of mortality and loss of independence in the community-living older population. J Am Geriatr Soc. 2000 May; 48(5):493-8.

5. Cruz-Jimenez M. Normal changes in gait and mobility problems in the elderly. Phys Med Rehabil Clin N Am. 2017 Nov; 28(4):713-25.

6. Brown CJ, Flood KL. Mobility limitation in the older patient: a clinical review. JAMA. 2013 Sep; 310(11):1168-77.

7. Rehabilitation Measures Database, Timed Up and Go [Internet]. 2013 [cited 2019 Sep]. Available from: https://www.sralab.org/ rehabilitation-measures/timed-and-go

8. Podsiadlo D, Richardson S. The timed "Up \& Go": a test of basic functional mobility for frail elderly persons. J Am Geriatr Soc. 1991 Feb; 39(2):142-8.
9. Academy of Neurologic Physical Therapy, Academy of Neurologic PT Outcome Measures Recommendations [Internet]. No date [cited 2019 Sep]. Available from: http://www.neuropt.org/practice-resources/ neurology-section-outcome-measures-recommendations

10. Portney LG, Watkins MP. Foundations of clinical research, 3rd ed. New Jersey: Pearson Education, Inc.; 2009.

11. Vereeck L, Wuyts F, Truijen S, Van de Heyning P. Clinical assessment of balance: normative data, and gender and age effects. Int J Audiol. $2008 \mathrm{Feb} ; 47(2): 67-75$

12. Bohannon RW. Reference values for the timed up and go test: a descriptive meta-analysis. J Geriatr Phys Ther. 2006; 29(2):64-8.

13. Steffen TM, Hacker TA, Mollinger L. Age- and gender-related test performance in community-dwelling elderly people: Six-Minute Walk Test, Berg Balance Scale, Timed Up \& Go Test, and gait speeds. Phys Ther. 2002 Feb; 82(2):128-37.

14. Lusardi MM, Pellecchia GL, Schulman M. Functional performance in community living older adults. J Geriatr Phys Ther. 2003 Dec 26(3):14-22.

15. Thaweewannakij T, Wilaichit S, Chuchot R, Yuenyong Y, Saengsuwan J, Siritaratiwat W, et al. Reference values of physical performance in Thai elderly people who are functioning well and dwelling in the community. Phys Ther. 2013 Oct; 93(10):1312-20.

16. Rahman NIA, Dawal SZM, Yusoff N, Kamil NSM. Anthropometric measurements among four Asian countries in designing sitting and standing workstations. Sādhanā. 2018; 43:10.

17. Patla AE, Shumway-Cook A. Dimensions of mobility: defining the complexity and difficulty associated with community mobility. J Aging Phys Act. 1999; 7:7-19.

18. Ibrahim A, Singh DKA, Shahar S. 'Timed Up and Go' test: Age, gender and cognitive impairment stratified normative values of older adults. PLoS One. 2017 Oct; 12(10):e0185641.

19. Lunar FR, Marquez JP, Quianzon FK, Policarpio BJ, Santelices LA, Velasco MK, et al. Mobility performance among community-dwelling older Filipinos who lived in urban and rural settings: A preliminary study. Hong Kong Physiother J. 2019 Dec; 39(2):91-9.

20. Morala DT, Shiomi T, Maruyama H. Factors associated with the functional status of community dwelling elderly. J Geriatr Phys Ther. 2006; 29(3):101-6.

21. Herman T, Giladi N, Hausdorff JM. Properties of the 'timed up and go' test: more than meets the eye. Gerontology. 2011; 57(3):203-10. 